\title{
Learning Dynamic Context Augmentation for Global Entity Linking
}

\author{
Xiyuan Yang ${ }^{1}$, Xiaotao $\mathrm{Gu}^{2}$, Sheng Lin $^{1}$, Siliang Tang ${ }^{1 *}$, Yueting Zhuang ${ }^{1}$, \\ Fei Wu ${ }^{1}$, Zhigang Chen ${ }^{3}$, Guoping $\mathrm{Hu}^{3}$ \& Xiang Ren $^{4}$ \\ ${ }^{1}$ Zhejiang University, ${ }^{2}$ University of Illinois at Urbana Champaign \\ ${ }^{3}$ iFLYTEK Research, ${ }^{4}$ University of Southern California \\ \{yangxiyuan, shenglin, siliang, yzhuang, wufei\}ezju.edu.cn, \\ xiaotao2@illinois.edu, \{zgchen, gphu\}@iflytek.com, \\ xiangren@usc.edu
}

\begin{abstract}
Despite of the recent success of collective entity linking (EL) methods, these "global" inference methods may yield sub-optimal results when the "all-mention coherence" assumption breaks, and often suffer from high computational cost at the inference stage, due to the complex search space. In this paper, we propose a simple yet effective solution, called Dynamic Context Augmentation (DCA), for collective EL, which requires only one pass through the mentions in a document. DCA sequentially accumulates context information to make efficient, collective inference, and can cope with different local EL models as a plugand-enhance module. We explore both supervised and reinforcement learning strategies for learning the DCA model. Extensive experiments ${ }^{1}$ show the effectiveness of our model with different learning settings, base models, decision orders and attention mechanisms.
\end{abstract}

\section{Introduction}

Linking mentions of entities in text to knowledge base entries (i.e., entity linking, or EL) is critical to understanding and structuring text corpora. In general, EL is approached by first obtaining candidate entities for each mention, and then identifying the true referent among the candidate entities. Prior distribution and local contexts, either in the form of hand-crafted features (Ratinov et al., 2011; Shen et al., 2015) or dense embeddings (He et al., 2013; Nguyen et al., 2016; Francis-Landau et al., 2016), play key roles in distinguishing different candidates. However, in many cases, local features can be too sparse to provide sufficient information for disambiguation.

To alleviate this problem, various collective EL models have been proposed to globally optimize

\footnotetext{
${ }^{*}$ Corresponding author.

${ }^{1}$ Code and data is available at https://github.com/YoungXiyuan/DCA
}

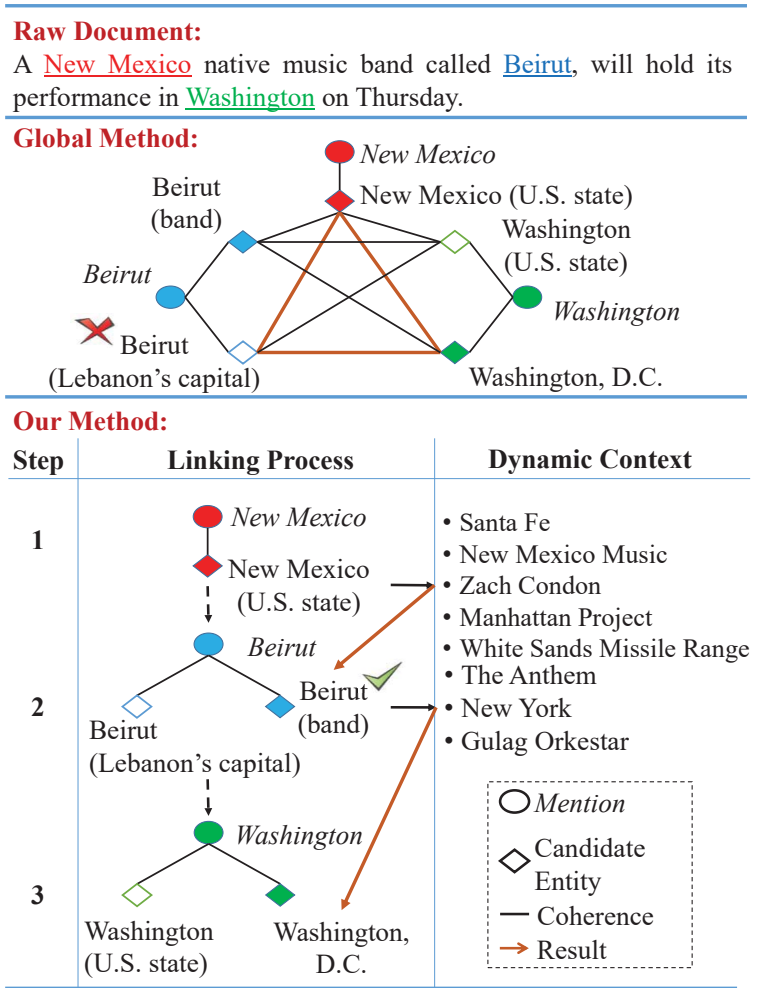

Figure 1: An Illustration of the Dynamic Context Augmentation process. A traditional global EL model jointly optimizes the linking configuration after iterative calculations over all mentions, which is computationally expensive. In contrast, the DCA process only requires one pass of the document to accumulate knowledge from previously linked mentions to enhance fast future inference.

the inter-entity coherence between mentions in the same document (Hoffart et al., 2011; Cheng and Roth, 2013; Nguyen et al., 2014; Alhelbawy and Gaizauskas, 2014; Pershina et al., 2015). Despite of their success, existing global EL models try to optimize the entire linking configuration of all mentions, with extra assumptions of either allmention coherence or pairwise coherence (Phan et al., 2018). Such assumptions are against human intuitions, as they imply that no inference can 
be made until all mentions in a document have been observed. Also, there usually exists a tradeoff between accuracy and efficiency: state-of-theart collective/global models suffer from high time complexity. From the perspective of computational efficiency, optimal global configuration inference is NP-hard. Approximation methods, such as loopy belief propagation (Ganea and Hofmann, 2017) or iterative substitutions (Shen et al., 2015), are still computationally expensive due to the huge hypothesis space, and thus can hardly be scaled to handle large corpus. Many previous works have discussed the urgent needs of more efficient linking system for production, both in time complexity (Hughes et al., 2014) and memory consumption (Blanco et al., 2015).

In this paper, we propose a simple yet effective Dynamic Context Augmentation (DCA) process to incorporate global signal for EL. As Figure 1 shows, in contrast to traditional global models, DCA only requires one pass through all mentions to achieve comparable linking accuracy. The basic idea is to accumulate knowledge from previously linked entities as dynamic context to enhance later decisions. Such knowledge come from not only the inherent properties (e.g., description, attributes) of previously linked entities, but also from their closely related entities, which empower the model with important associative abilities. In real scenarios, some previously linked entities may be irrelevant to the current mention. Some falsely linked entities may even introduce noise. To alleviate error propagation, we further explore two strategies: (1) soft/hard attention mechanisms that favour the most relevant entities; (2) a reinforcement learning-based ranking model, which proves to be effective as reported in other information extraction tasks.

Contributions. The DCA model forms a new linking strategy from the perspective of data augmentation and thus can serve as a plug-andenhance module of existing linking models. The major contributions of this work are as follows: (1) DCA can introduce topical coherence into local linking models without reshaping their original designs or structures; (2) Comparing to global EL models, DCA only requires one pass through all mentions, yielding better efficiency in both training and inference; (3) Extensive experiments show the effectiveness of our model under different learning settings, base models, decision orders and attention mechanisms.

\section{Background}

\subsection{Problem Definition}

Given a set of entity mentions $\mathcal{M}=\left\{m_{1}, \ldots, m_{T}\right\}$ in corpus $\mathcal{D}$, Entity Linking aims to link each mention $m_{t}$ to its corresponding gold entity $e_{t}^{*}$. Such a process is usually divided into two steps: Candidate generation first collects a set of possible (candidate) entities $\mathcal{E}_{t}=\left\{e_{t}^{1}, \ldots, e_{t}^{\left|\mathcal{E}_{t}\right|}\right\}$ for $m_{t}$; Candidate ranking is then applied to rank all candidates by likelihood. The linking system selects the top ranked candidate as the predicted entity $\hat{e}_{t}$. The key challenge is to capture high-quality features of each entity mention for accurate entity prediction, especially when local contexts are too sparse to disambiguate all candidates.

We build our DCA model based on two existing local EL models. In this section, we first introduce the architecture of the base models, then present the proposed DCA model under the standard supervised learning framework. Since the DCA process can be naturally formed as a sequential decision problem, we also explore its effectiveness under the Reinforcement Learning framework. Detailed performance comparison and ablation studies are reported in Section 6.

\subsection{Local Base Models for Entity Linking}

We apply the DCA process in two popular local models with different styles: the first is a neural attention model named ETHZ-Attn (Ganea and Hofmann, 2017), the other is the BerkeleyCNN (Francis-Landau et al., 2016) model which is made up of multiple convolutional neural networks $(\mathrm{CNN})$.

ETHZ-Attn. For each mention $m_{t}$ and a candidate $e_{t}^{j} \in \mathcal{E}_{t}$, three local features are considered: (1) Mention-entity Prior $\hat{P}\left(e_{t}^{j} \mid m_{t}\right)$ is the empirical distribution estimated from massive corpus (e.g.Wikipedia); (2) Context Similarity $\Psi_{C}\left(m_{t}, e_{t}^{j}\right)$ measures the textual similarity between $e_{t}^{j}$ and the local context of $m_{t}$; (3) Type Similarity $\Psi_{T}\left(m_{t}, e_{t}^{j}\right)$ considers the similarity between the type of $e_{t}^{j}$ and contexts around $m_{t} . \hat{P}\left(e_{t}^{j} \mid m_{t}\right)$ and $\Psi_{C}\left(m_{t}, e_{t}^{j}\right)$ are calculated in the same way as (Ganea and Hofmann, 2017). For $\Psi_{T}\left(m_{t}, e_{t}^{j}\right)$, we first train a typing system proposed by (Xu and Barbosa, 2018) on AIDAtrain dataset, yielding 95\% accuracy on AIDA-A dataset. In the testing phase, the typing system 
predicts the probability distribution over all types (PER, GPE, ORG and UNK) for $m_{t}$, and outputs $\Psi_{T}\left(m_{t}, e_{t}^{j}\right)$ for each candidate accordingly. All local features are integrated by a two-layer feedforward neural network with 100 hidden units, as described in (Ganea and Hofmann, 2017).

Berkeley-CNN. The only difference between ETHZ-Attn and Berkeley-CNN is that, this model utilizes CNNs at different granularities to capture context similarity $\Psi_{C}\left(m_{t}, e_{t}^{j}\right)$ between a mention's context and its target candidate entities.

\section{Dynamic Context Augmentation}

As Figure 1 demonstrates, the basic idea of DCA is to accumulate knowledge from previously linked entities as dynamic context to enhance later decisions. Formally, denote the list of previously linked entities as $S_{t}=\left\{\hat{e}_{1}, \ldots, \hat{e}_{t}\right\}$, where each $\hat{e}_{i}$ is represented as an embedding vector. The augmented context can be represented by accumulated features of all previous entities and their neighbors (e.g. by averaging their embeddings, in the simplest way). In actual scenarios, some entities in $S_{t}$ are irrelevant, if not harmful, to the linking result of $m_{t+1}$. To highlight the importance of relevant entities while filtering noises, we also try to apply a neural attention mechanism on dynamic contexts (Figure 2). For mention $m_{t+1}$, candidates that are more coherent with $S_{t}$ are preferred. More specifically, we calculate the relevance score for each $\hat{e}_{i} \in S_{t}$ as

$$
u\left(\hat{e}_{i}\right)=\max _{e_{t+1}^{j} \in \mathcal{E}_{t+1}} e_{t+1}^{j}{ }^{\top} \cdot A \cdot \hat{e}_{i},
$$

where $A$ is a parameterized diagonal matrix. Top $K$ entities in $S_{t}$ are left to form dynamic context while the others are pruned. The relevance scores are transformed to attention weights with

$$
a\left(\hat{e}_{i}\right)=\frac{\exp \left[u\left(\hat{e}_{i}\right)\right]}{\sum_{\hat{e}_{j} \in S_{t}} \exp \left[u\left(\hat{e}_{j}\right)\right]} .
$$

Thus, we can define a weighted coherence score between $e_{t+1}^{j} \in \mathcal{E}_{t+1}$ and $S_{t}$ as

$$
\Phi\left(e_{t+1}^{j}, S_{t}\right)=\sum_{\hat{e}_{i} \in S_{t}} a\left(\hat{e}_{i}\right) \cdot e_{t+1}^{j}{ }^{\top} \cdot R \cdot \hat{e}_{i},
$$

where $R$ is a learnable diagonal matrix. Such a coherence score will be later incorporated in the final representation of $e_{t+1}^{j}$.

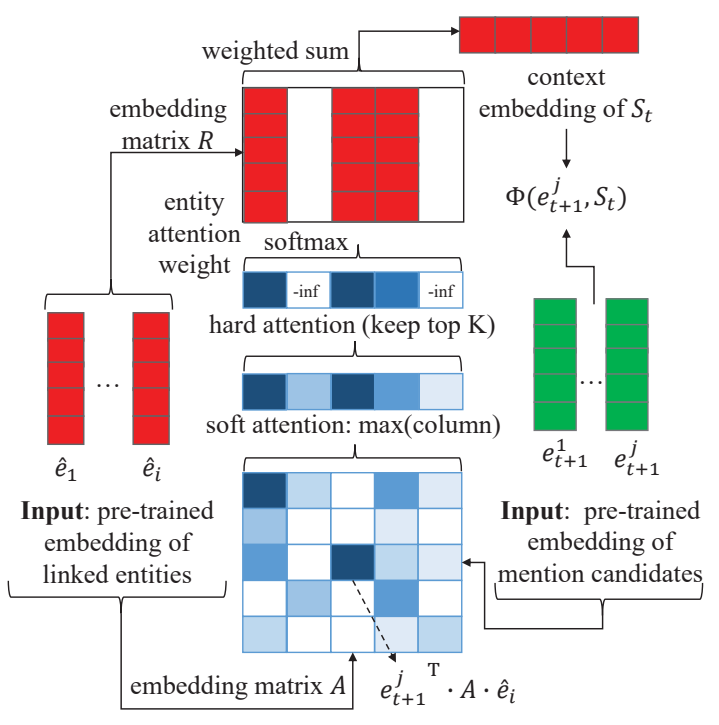

Figure 2: Neural attention mechanism on the dynamic context. The soft attention module assigns higher weights to entities that are more relevant to the target mention. The hard attention module only considers top $K$ entities as dynamic contexts.

To empower the linking model with associative ability, aside from previously linked entities, we also incorporate entities that are closely associated with entities in $S_{t}$. Specifically, for each $\hat{e}_{i} \in S_{t}$, we collect its neighborhood $\mathcal{N}\left(\hat{e}_{i}\right)$ consisting of Wikipedia entities that have inlinks pointing to $\hat{e}_{i}$. Denoting $S_{t}^{\prime}$ as the union of $\left\{\mathcal{N}\left(\hat{e}_{i}\right) \mid \hat{e}_{i} \in S_{t}\right\}$, we define a similar weighted coherence score between $e_{t+1}^{j} \in \mathcal{E}_{t+1}$ and $S_{t}^{\prime}$ as

$$
\Phi^{\prime}\left(e_{t+1}^{j}, S_{t}^{\prime}\right)=\sum_{\hat{e}_{i} \in S_{t}^{\prime}} a^{\prime}\left(\hat{e}_{i}\right) \cdot e_{t+1}^{j}{ }^{\top} \cdot R^{\prime} \cdot \hat{e}_{i},
$$

where $a^{\prime}$ is defined similarly to $a$, and $R^{\prime}$ is a learnable diagonal matrix. The final representation $\overrightarrow{h_{0}}\left(m_{t+1}, e_{t+1}^{j}\right)$ is the concatenation of $\Phi\left(e_{t+1}^{j}, S_{t}\right), \Phi^{\prime}\left(e_{t+1}^{j}, S_{t}^{\prime}\right), \Psi_{T}\left(m_{t}, e_{t+1}^{j}\right)$, $\Psi_{C}\left(m_{t}, e_{t+1}^{j}\right)$ and $\log \hat{P}\left(e_{t+1}^{j} \mid m_{t+1}\right)$.

\section{Model Learning for DCA}

In this section, we explore different learning strategies for the linking model. Specifically, we present a Supervised Learning model, where the model is given all gold entities for training, and a Reinforcement Learning model, where the model explores possible linking results by itself in a longterm planning task.

\subsection{Supervised Ranking Method}

Given a mention-candidate pair $\left(m_{t}, e_{t}^{j}\right)$, the ranking model parameterized by $\theta$ accepts the feature 
vector $\overrightarrow{h_{0}}\left(m_{t}, e_{t}^{j}\right)$ as input, and outputs the probability $P_{\theta}\left(e_{t}^{j} \mid m_{t}\right)$. In this work, we use a two-layer feedforward neural network as the ranking model. We apply the max-margin loss as

$$
\mathcal{L}_{\theta}=\sum_{D \in \mathcal{D}} \sum_{m_{t} \in D} \sum_{e_{t}^{j} \in \mathcal{E}_{t}} g_{\theta}\left(e_{t}^{j}, m_{t}\right),
$$

$g_{\theta}\left(e_{t}^{j}, m_{t}\right)=\max \left(0, \gamma-P_{\theta}\left(e_{t}^{*} \mid m_{t}\right)+P_{\theta}\left(e_{t}^{j} \mid m_{t}\right)\right)$.

The learning process is to estimate the optimal parameter such that $\theta^{*}=\arg \min _{\theta} \mathcal{L}_{\theta}$.

Note that, in the Supervised Ranking model, dynamic contexts are provided by previous gold entities: $S_{t}=\left\{e_{1}^{*}, \ldots, e_{t}^{*}\right\}, S_{t}^{\prime}=\bigcup_{i=1}^{t} \mathcal{N}\left(e_{i}^{*}\right)$. In the testing phase, however, we do not have access to gold entities. Wrongly linked entities can introduce noisy contexts to future linking steps. To consider such long-term influences, we introduce an alternative Reinforcement Learning model in the next section.

\subsection{Reinforcement Learning Method}

Naturally, the usage of dynamic context augmentation forms a sequential decision problem, as each linking step depends on previous linking decisions. Correct linking results provide valuable information for future decisions, while previous mistakes can lead to error accumulation. Reinforcement Learning (RL) algorithms have proven to be able to alleviate such accumulated noises in the decision sequence in many recent works (Narasimhan et al., 2016; Feng et al., 2018). In this work, we propose an RL ranking model for DCA-enhanced entity linking.

Agent: The Agent is a candidate ranking model that has a similar architecture to (Clark and Manning, 2016), aiming to output the action preference $H_{\theta}\left(S_{t-1}, S_{t-1}^{\prime}, A_{t}^{j}\right)$ of each linking action $A_{t}^{j}=\left(m_{t} \rightarrow e_{t}^{j}\right)$. It is a 2-layer feedforward neural network with following components:

Input Layer: For each $\left(m_{t}, e_{t}^{j}\right)$ pair, DCARL extracts context-dependent features from $S_{t-1}, S_{t-1}^{\prime}$, and concatenates them with other context-independent features to produce an $I$ dimensional input vector $\overrightarrow{h_{0}}\left(m_{t}, e_{t}^{j}\right)$.

Hidden Layers: Let $\operatorname{Drop}(\vec{x})$ be the dropout operation (Srivastava et al., 2014) and $\operatorname{Re} L U(\vec{x})$ be the rectifier nonlinearity (Nair and Hinton, 2010). So the output $\vec{h}_{1}$ of the hidden layer is defined as:

$$
\vec{h}_{1}=\operatorname{Drop}\left(\operatorname{Re} L U\left(\vec{W}_{1} \cdot \vec{h}_{0}+\vec{b}_{1}\right)\right),
$$

where $\vec{W}_{1}$ is a $H_{1} \times I$ weight matrix.

Output Layers: This scoring layer is also fully connected layer of size 1 .

$$
\vec{h}_{2}=\vec{W}_{2} \cdot \vec{h}_{1}+\vec{b}_{2},
$$

where $\vec{W}_{2}$ is a $1 \times H_{1}$ weight matrix. In the end, all action preference would be normalized together using an exponential softmax distribution, getting their action probabilities $\pi_{\theta}\left(A_{t}^{j} \mid S_{t-1}, S_{t-1}^{\prime}\right)$ :

According to policy approximating methods, the best approximate policy may be stochastic. So we randomly sample the actions based on the softmax distribution during the training time, whereas deliberately select the actions with the highest ranking score at the test time.

Reward. The reward signals are quite sparse in our framework. For each trajectory, the Agent can only receive a reward signal after it finishes all the linking actions in a given document. Therefore the immediate reward of action $t, R_{t}=0$, where $0 \leq t<T$, and $R_{T}=-\left(\left|\mathbb{M}_{e}\right| / T\right)$, where $T$ is total number of mentions in the source document, and $\left|\mathbb{M}_{e}\right|$ is the number of incorrectly linked mentions. Then the value $G_{t}$ (expected reward) of each previous state $S_{t}$ can be retraced back with a discount factor $\rho$ according to $R_{T}$ :

$$
G_{t}=\rho^{T-t} R_{T}
$$

To maximize the expected reward of all trajectories, the Agent utilizes the REINFORCE algorithm (Sutton and Barto, 1998) to compute Monte Carlo policy gradient over all trajectories, and perform gradient ascent on its parameters:

$$
\theta \leftarrow \theta+\alpha \sum_{t} G_{t} \nabla_{\theta} \ln \pi_{\theta}\left(A_{t}^{j} \mid S_{t-1}, S_{t-1}^{\prime}\right)
$$

In following sections, to fully investigate the effectiveness of the proposed method, we report and compare the performances of both the Supervisedlearning model and the Reinforcement-learning model.

\section{Analysis of Computational Complexity}

For each document $D$, the train and inference of the global EL models are heavily relied on the inter-entity coherence graph $\Phi_{g}$. Many studies (Ratinov et al., 2011; Globerson et al., 2016; Yamada et al., 2016; Ganea and Hofmann, 2017; Le and Titov, 2018) obtain $\Phi_{g}$ by calculating all 


\begin{tabular}{lcccc}
\hline Dataset & $\begin{array}{c}\# \\
\text { mention }\end{array}$ & $\begin{array}{c}\# \\
\text { doc }\end{array}$ & $\begin{array}{c}\text { Mentions } \\
\text { per doc }\end{array}$ & $\begin{array}{c}\text { Gold } \\
\text { recall }\end{array}$ \\
\hline AIDA-train & 18448 & 946 & 19.5 & - \\
AIDA-A & 4791 & 216 & 22.1 & 97.3 \\
AIDA-B & 4485 & 231 & 19.4 & 98.3 \\
\hline MSNBC & 656 & 20 & 32.8 & 98.5 \\
AQUAINT & 727 & 50 & 14.5 & 94.2 \\
ACE2004 & 257 & 36 & 7.1 & 90.6 \\
CWEB & 11154 & 320 & 34.8 & 91.1 \\
WIKI & 6821 & 320 & 21.3 & 92.4 \\
\hline
\end{tabular}

Table 1: Dataset Statistics. Gold recall is the percentage of mentions for which the candidate entities contain the ground truth entity.

pairwise scores between two arbitrary elements $e_{i}^{x}$ and $e_{j}^{y}$ sampled independently from candidate sets $\mathcal{E}_{i}$ and $\mathcal{E}_{j}$ in the given document. It is obvious that $\Phi$ is intractable, and the computational complexity of $\Phi_{g}$ is

$$
\mathcal{O}\left(\Phi_{g}\right)=\mathcal{O}\left(\sum_{i=1}^{T} \sum_{j=1, j \neq i}^{T} \sum_{e_{i} x \in \mathcal{E}_{i}}^{\left|\mathcal{E}_{i}\right|} \sum_{e_{j}}^{\left|\mathcal{E}_{j}\right|} \Phi\left(e_{i}{ }^{x}, e_{j}{ }^{y}\right)\right)
$$

, where $\Phi\left(e_{i}^{x}, e_{j}^{y}\right)$ is a learnable score function. Thus, $\mathcal{O}\left(\Phi_{g}\right)$ is approximate to $\mathcal{O}\left(T^{2} \times|\mathcal{E}|^{2} \times \mathcal{I}\right)$, where $|\mathcal{E}|$ is the average number of candidates per mention and $\mathcal{I}$ is the unit cost of pairwise function $\Phi$. In order to reduce $\mathcal{O}\left(\Phi_{g}\right)$, most previous models (Hoffart et al., 2011; Ganea and Hofmann, 2017; Le and Titov, 2018; Fang et al., 2019) have to hard prune their candidates into an extremely small size (e.g. $|\mathcal{E}|=5$ ). This will reduce the gold recall of candidate sets and also unsuitable for large scale production (e.g. entity disambiguation for dynamic web data like Twitter).

In contrast, the computational complexity of our model is $\mathcal{O}(T \times|\mathcal{E}| \times \mathcal{I} \times K)$, where $K$ is the key hyper-parameter described in Section 3 and is usually set to a small number. This indicates the response time of our method grow linearly as a function of $T \times|\mathcal{E}|$.

\section{Experiment}

\subsection{Experiment Setup}

Datasets. Following our predecessors, we train and test all models on the public and widely used AIDA CoNLL-YAGO dataset (Hoffart et al., 2011). The target knowledge base is Wikipedia. The corpus consists of 946 documents for training, 216 documents for development and 231 documents for testing (AIDA-train/A/B respectively).

\begin{tabular}{lc}
\hline System & In-KB acc. (\%) \\
\hline AIDA-light (Nguyen et al., 2014) & 84.8 \\
WNED (Guo and Barbosa, 2016) & 89.0 \\
Global-RNN (Nguyen et al., 2016) & 90.7 \\
MulFocal-Att (Globerson et al., 2016) & 91.0 \\
Deep-ED (Ganea and Hofmann, 2017) & 92.22 \\
Ment-Norm (Le and Titov, 2018) & 93.07 \\
\hline Prior $(p(e \mid m))$ (Ganea and Hofmann, 2017) & 71.51 \\
\hline Berkeley-CNN (Sec. 2.2) & 84.21 \\
Berkeley-CNN + DCA-SL & $92.72 \pm 0.3$ \\
Berkeley-CNN + DCA-RL & $92.37 \pm 0.1$ \\
ETHZ-Attn (Sec. 2.2) & 90.88 \\
ETHZ-Attn + DCA-SL & $\mathbf{9 4 . 6 4} \pm 0.2$ \\
ETHZ-Attn + DCA-RL & $93.73 \pm 0.2$ \\
\hline
\end{tabular}

Table 2: In-domain Performance Comparison on the AIDA-B Dataset. For our method we show 95\% confidence intervals obtained over 5 runs. DCA-based models achieve the best reported scores on this benchmark.

To evaluate the generalization ability of each model, we apply cross-domain experiments following the same setting in (Ganea and Hofmann, 2017; Le and Titov, 2018; Yang et al., 2018). Models are trained on AIDA-train, and evaluated on five popular public datasets: AQUAINT (Milne and Witten, 2008), MSNBC (Cucerzan, 2007), ACE2004 (Ratinov et al., 2011), CWEB (Guo and Barbosa, 2016) and WIKI (Guo and Barbosa, 2016). The statistics of these datasets are available in Table 1. In the candidate generation step, we directly use the candidates provided by the Ment-Norm system (Le and Titov, 2018) ${ }^{2}$, and their quality is also listed in Table 1.

Compared Methods. We compare our methods with following existing systems that report state-of-the-art results on the test datasets: AIDAlight (Nguyen et al., 2014) uses a kind of twostage collective mapping algorithm and designs several domain or category related coherence features. WNED (Guo and Barbosa, 2016) applies random walks on carefully built disambiguation graphs and uses a greedy, iterative and global disambiguation algorithm based on Information Theory. Global-RNN (Nguyen et al., 2016) develops a framework based on convolutional neural networks and recurrent neural networks to simultaneously model the local and global features. MulFocal-Att (Globerson et al., 2016) adopts a coherence model with a multi-focal attention mechanism. Deep-ED (Ganea and Hofmann, 2017) leverages learned neural representa-

\footnotetext{
${ }^{2}$ https://github.com/lephong/mulrel-nel
} 


\begin{tabular}{lccccc}
\hline System & MSBNC & AQUAINT & ACE2004 & CWEB & WIKI \\
\hline AIDA (Hoffart et al., 2011) & 79 & 56 & 80 & 58.6 & 63 \\
GLOW (Ratinov et al., 2011) & 75 & 83 & 82 & 56.2 & 67.2 \\
RI (Cheng and Roth, 2013) & 90 & $\mathbf{9 0}$ & 86 & 67.5 & 73.4 \\
WNED (Guo and Barbosa, 2016) & 92 & 87 & 88 & 77 & $\mathbf{8 4 . 5}$ \\
Deep-ED (Ganea and Hofmann, 2017) & 93.7 & 88.5 & 88.5 & $\mathbf{7 7 . 9}$ & 77.5 \\
Ment-Norm (Le and Titov, 2018) & 93.9 & 88.3 & 89.9 & 77.5 & 78.0 \\
\hline Prior ( $($ (e|m)) (Ganea and Hofmann, 2017) & 89.3 & 83.2 & 84.4 & 69.8 & 64.2 \\
\hline Berkeley-CNN (Section 2.2) & 89.05 & 80.55 & 87.32 & 67.97 & 60.27 \\
Berkeley-CNN + DCA-SL & $93.38 \pm 0.2$ & $85.63 \pm 0.3$ & $88.73 \pm 0.3$ & $71.01 \pm 0.1$ & $72.55 \pm 0.2$ \\
Berkeley-CNN + DCA-RL & $93.65 \pm 0.2$ & $88.53 \pm 0.3$ & $89.73 \pm 0.4$ & $72.66 \pm 0.4$ & $73.98 \pm 0.2$ \\
ETHZ-Attn (Section 2.2) & 91.97 & 84.06 & 86.92 & 70.07 & 74.37 \\
ETHZ-Attn + DCA-SL & $\mathbf{9 4 . 5 7} \pm 0.2$ & $87.38 \pm 0.5$ & $89.44 \pm 0.4$ & $73.47 \pm 0.1$ & $78.16 \pm 0.1$ \\
ETHZ-Attn + DCA-RL & $93.80 \pm 0.0$ & $88.25 \pm 0.4$ & $\mathbf{9 0 . 1 4} \pm 0.0$ & $75.59 \pm 0.3$ & $78.84 \pm 0.2$ \\
\hline
\end{tabular}

Table 3: Performance Comparison on Cross-domain Datasets using F1 score (\%). The best results are in bold. Note that our own results all retain two decimal places. Other results with uncertain amount of decimal places are directly retrieved from their original paper.

tions, and uses a deep learning model combined with a neural attention mechanism and graphical models. Ment-Norm (Le and Titov, 2018) improving the Deep-ED model by modeling latent relations between mentions.

For a fair comparison with prior work, we use the same input as the WNED, Deep-ED and Ment-Norm (models proposed after 2016), and report the performance of our model with both Supervised Learning (DCA-SL) and Reinforcement Learning (DCA-RL). We won't compare our models with the RLEL (Fang et al., 2019) which is a deep reinforcement learning based LSTM model. There are two reasons: 1) RLEL uses optimized candidate sets with smaller candidate size and higher gold recall than ours and the listed baselines. 2) RLEL uses additional training set from Wikipedia data. (Fang et al., 2019) doesn't release either their candidate sets or updated training corpus, so the comparison with their work would be unfair for us.

Hyper-parameter Setting. We coarsely tune the hyper-parameters according to model performance on AIDA-A. We set the dimensions of word embedding and entity embedding to 300 , where the word embedding and entity embedding are publicly released by (Pennington et al., 2014) and (Ganea and Hofmann, 2017) respectively. Hyper-parameters of the best validated model are: $K=7, I=5, H_{1}=100$, and the probability of dropout is set to 0.2. Besides, the rank margin $\gamma=0.01$ and the discount factor $\rho=0.9$. We also

\begin{tabular}{c|cc}
\hline \multirow{2}{*}{ System } & \multicolumn{2}{|c}{ In-KB acc. $(\%)$} \\
\cline { 2 - 3 } & SL & RL \\
\hline ETHZ-Attn (Section 2.2) & 90.88 & - \\
ETHZ-Attn + 1-hop DCA & 93.69 & 93.20 \\
ETHZ-Attn + 2-hop DCA & 94.47 & 93.76 \\
\hline
\end{tabular}

Table 4: Ablation Study on Neighbor Entities. We compare the performance of DCA with or without neighbor entities (i.e., 2-hop vs. 1-hop).

regularize the Agent model as adopted in (Ganea and Hofmann, 2017) by constraining the sum of squares of all weights in the linear layer with MaxNorm $=4$. When training the model, we use Adam (Kingma and Ba, 2014) with learning rate of 2e-4 until validation accuracy exceeds $92.8 \%$, afterwards setting it to $5 \mathrm{e}-5$.

\subsection{Overall Performance Comparison}

Starting with an overview of the end-task performance, we compare DCA (using SL or RL) with several state-of-the-art systems on in-domain and cross-domain datasets. We follow prior work and report in-KB accuracy for AIDA-B and micro F1 scores for the other test sets.

Table 2 summarizes results on the AIDA$B$ dataset, and shows that DCA-based models achieve the highest in-KB accuracy and outperforms the previous state-of-the-art neural system by near $1.6 \%$ absolute accuracy. Moreover, compared with the base models, dynamic context augmentation significantly improve absolute in-KB accuracy in models Berkeley-CNN (more than 
In-domain Dataset

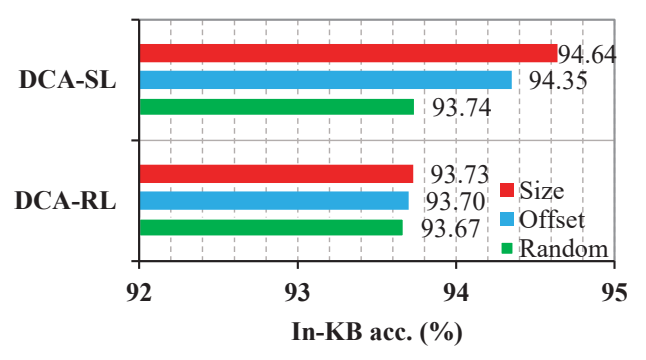

Cross-domain Dataset

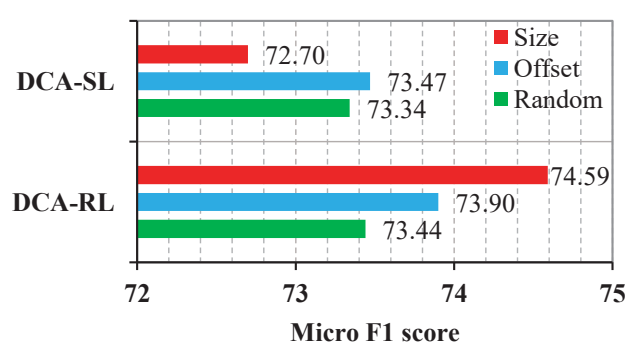

AIDA-B

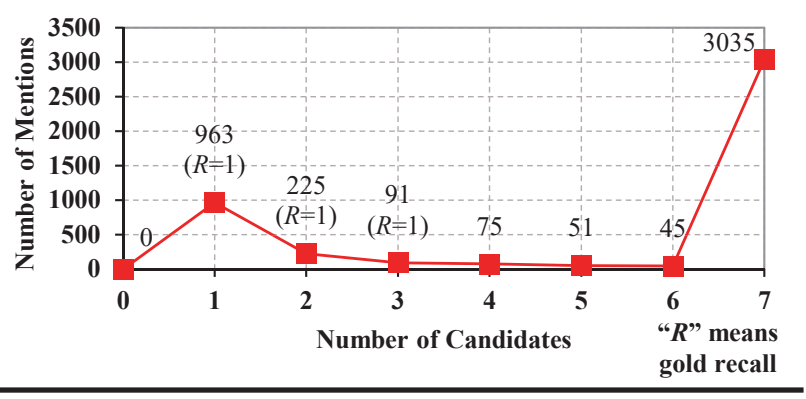

CWEB

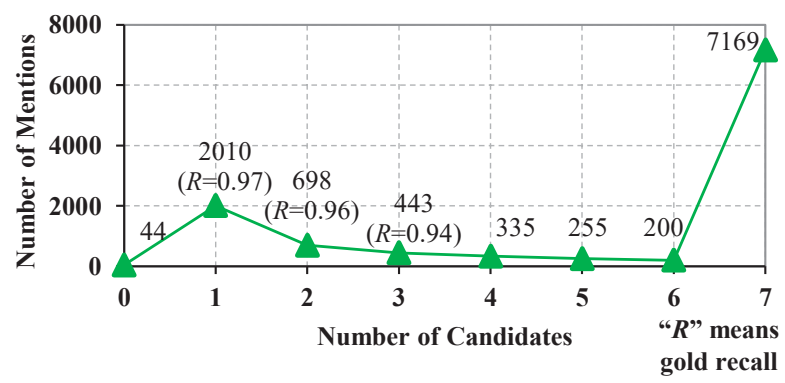

Figure 3: Ablation Study on Different Decision Orders. We test on both in-domain (AIDA-B) and cross-domain (CWEB) datasets, using ETHZ-Attn as the local model.

8\%) and ETHZ-Attn (3.3\% on average). Note that, our DCA model outperforms existing global models with the same local model (Global-RNN uses Berkeley-CNN as base model, Deep-ED and Ment-Norm use ETHZ-Attn as the local model).

Table 3 shows the results on the five crossdomain datasets. As shown, none of existing methods can consistently win on all datasets. DCA-based models achieve state-of-the-art performance on the MSBNC and the ACE2004 dataset. On remaining datasets, DCA-RL achieves comparable performance with other complex global models. In addition, RL-based models show on average $1.1 \%$ improvement on F1 score over the SL-based models across all the crossdomain datasets. At the same time, DCA-based methods are much more efficient, both in time complexity and in resource requirement. Detailed efficiency analysis will be presented in following sections.

\subsection{Performance Analysis}

1. Impact of decision order. As the DCA model consecutively links and adds all the mentions in a document, the linking order may play a key role in the final performance. In this work, we try three different linking orders: Offset links all mentions by their natural orders in the original document;

\begin{tabular}{c|cc}
\hline \multirow{2}{*}{ System } & \multicolumn{2}{|c}{ In-KB acc. (\%) } \\
\cline { 2 - 3 } & SL & RL \\
\hline DCA + Average Sum & 94.05 & 93.56 \\
DCA + Soft Attention & 94.23 & 93.69 \\
DCA + Soft\&Hard Attention & 94.47 & 93.76 \\
\hline
\end{tabular}

Table 5: Study on Different Attention Mechanisms on the Dynamic Context. Results (using ETHZ-Attn as local model) show that different attention mechanisms have similar impact on the performance.

Size first links mentions with smaller candidate sizes, as they tend to be easier to link; The baseline method is to link all mentions in a Random order. Figure 3 shows the performance comparison on the AIDA-B and the CWEB dataset. As shown, in general, Size usually leads to better performance than Offset and Random. However, the DCA-SL model shows poor performance on the CWEB dataset with Size order. This is mainly because the CWEB dataset is automatically generated rather than curated by human, and thus contains many noisy mentions. Some mentions in CWEB with less than three candidates are actually bad cases, where none of the candidates is the actual gold entity. Thus, such mentions will always introduce wrong information to the model, which leads to a worse performance. In contrast, the AIDA-B dataset does not have such situations. 


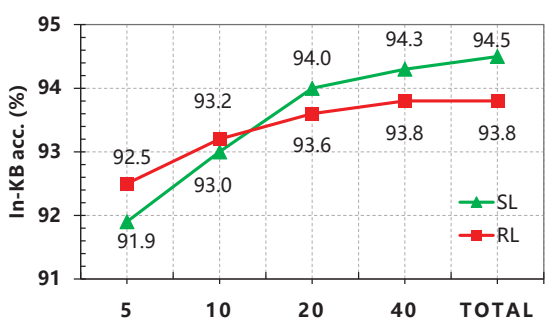

(a) Decision Length (the number of mentions)

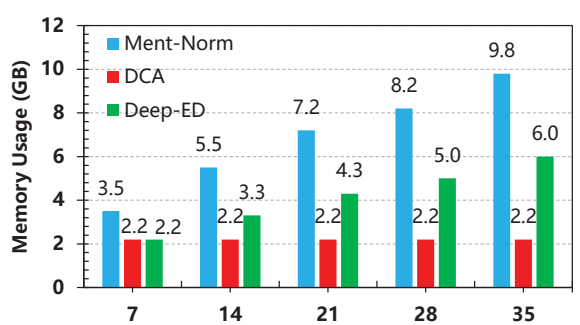

(b) The Number of Candidates per Mention

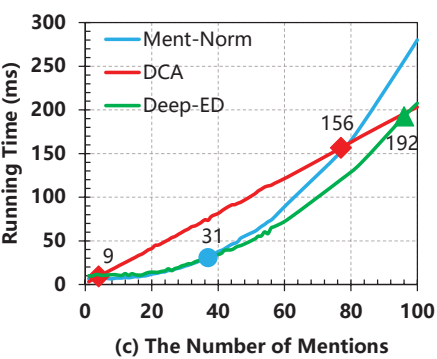

(c) The Number of Mentions

Figure 4: (a) In-KB accuracy as a function of decision length on AIDA-B dataset; (b) Memory usage as a function of the number of candidates on AIDA-B dataset; (c) Runtime cost (at inference time) as a function of the number of mentions. (each dot represents a document of AIDA-B with $|\mathcal{E}|=35$ ).

The DCA-RL model, however, still has strong performance on the CWEB dataset, which highlights its robustness to potential noises.

2. Effect of neighbor entities. In contrast to traditional global models, we include both previously linked entities and their close neighbors for global signal. Table 4 shows the effectiveness of this strategy. We observe that incorporating these neighbor entities (2-hop) significantly improve the performance (compared to 1-hop) by introducing more related information. And our analysis shows that on average $0.72 \%$ and $3.56 \%$ relative improvement of 2-hop DCA-(SL/RL) over 1-hop DCA-(SL/RL) or baseline-SL (without DCA) is statistically significant (with $\mathrm{P}$-value $<$ 0.005). This is consistent with our design of DCA.

\section{Study of different attention mechanisms.} Table 5 shows the performance comparison by replacing the attention module described in Section 3 with different variants. Average Sum treats all previously linked entities equally with a uniform distribution. Soft Attention skips the pruning step for entities with low weight scores. Soft\&Hard Attention stands for the strategy used in our model. It is obvious that the attention mechanism does show positive influence on the linking performance compared with Average Sum. Hard pruning brings slight further improvement.

4. Impact of decision length. As wrongly linked entities can introduce noise to the model, there exists a trade-off in DCA: involving more previous entities (longer historical trajectory) provides more information, and also more noise. Figure (4.a) shows how the performance of DCA changes with the number of previous entities involved. We observe that longer historical trajectories usually have a positive influence on the performance of DCA. The reason is that our attention mechanism could effectively assess and select relevant contexts for each entity mention on the fly, thus reducing potential noise.

\subsection{Analysis on Time Complexity}

As discussed in Sec. 5, the running time of a DCA enhanced model may rise linearly when the average number of candidates per mention (i.e., $|\mathcal{E}|$ ) increases, while the global EL model increases exponentially. To validate the theory we empirically investigate the scalability of DCA, and carefully select two global EL models Ment-Norm (Le and Titov, 2018) and Deep-ED (Ganea and Hofmann, 2017) as our baselines. The reason for this choice is that our final model shares the same local model as their models, which excludes other confounding factors like implementation details. As Figure (4.c) shows, when $|\mathcal{E}|$ increases, the running time of these two global EL models increases shapely, while our DCA model grows linearly. On the other hand, we also observed that the resources required by the DCA model are insensitive to $|\mathcal{E}|$. For example, as shown in Figure (4.b), the memory usage of Ment-Norm and Deep-ED significantly rises as more candidates are considered, while the DCA model remains a relatively low memory usage all the time. We also measure the power consumption of Ment-Norm and DCA models, and we find that the DCA model saves up to $80 \%$ of the energy consumption over the Ment-Norm, which is another advantage for large scale production.

\section{Related Work}

Local EL methods disambiguate each mention independently according to their local contexts (Yamada et al., 2016; Chen et al., 2017; Globerson et al., 2016; Raiman and Raiman, 2018). The per- 
formance is limited when sparse local contexts fail to provide sufficient disambiguation evidence.

To alleviate this problem, global EL models jointly optimize the entire linking configuration. The key idea is to maximize a global coherence/similarity score between all linked entities (Hoffart et al., 2011; Ratinov et al., 2011; Cheng and Roth, 2013; Nguyen et al., 2014; Alhelbawy and Gaizauskas, 2014; Pershina et al., 2015; Guo and Barbosa, 2016; Globerson et al., 2016; Ganea and Hofmann, 2017; Le and Titov, 2018; Yang et al., 2018; Fang et al., 2019; Xue et al., 2019). Despite of its significant improvement in accuracy, such global methods suffer from high complexity. To this end, some works try to relax the assumption of all-mention coherence, e.g. with pairwise coherence, to improve efficiency (Phan et al., 2018), but exact inference remains an NPhard problem. Approximation methods are hence proposed to achieve reasonably good results with less cost. (Shen et al., 2012) propose the iterative substitution method to greedily substitute linking assignment of one mention at a time that can improve the global objective. Another common practice is to use Loopy Belief Propagation for inference (Ganea and Hofmann, 2017; Le and Titov, 2018). Both approximation methods iteratively improve the global assignment, but are still computationally expensive with unbounded number of iterations. In contrast, the proposed DCA method only requires one pass through the document. Global signals are accumulated as dynamic contexts for local decisions, which significantly reduces computational complexity and memory consumption.

\section{Conclusions}

In this paper we propose Dynamic Context Augmentation as a plug-and-enhance module for local Entity Linking models. In contrast to existing global EL models, DCA only requires one pass through the document. To incorporate global disambiguation signals, DCA accumulates knowledge from previously linked entities for fast inference. Extensive experiments on several public benchmarks with different learning settings, base models, decision orders and attention mechanisms demonstrate both the effectiveness and efficiency of DCA-based models. The scalability of DCAbased models make it possible to handle largescale data with long documents. Related code and data has been published and may hopefully benefit the community.

\section{Acknowledgments}

This work has been supported in part by National Key Research and Development Program of China (SQ2018AAA010010), NSFC (No.61751209, U1611461), Zhejiang University-iFLYTEK Joint Research Center, Zhejiang University-Tongdun Technology Joint Laboratory of Artificial Intelligence, Hikvision-Zhejiang University Joint Research Center, Chinese Knowledge Center of Engineering Science and Technology (CKCEST), Engineering Research Center of Digital Library, Ministry of Education. Xiang Ren's research has been supported in part by National Science Foundation SMA 18-29268, DARPA MCS and GAILA, IARPA BETTER, Schmidt Family Foundation, Amazon Faculty Award, Google Research Award, Snapchat Gift and JP Morgan AI Research Award. Finally, we would like to thank Qi Zhang from Sogou, Inc. and all the collaborators in INK research lab for their constructive feedback on this work.

\section{References}

Ayman Alhelbawy and Robert Gaizauskas. 2014. Graph ranking for collective named entity disambiguation. In Proceedings of the 52nd Annual Meeting of the Association for Computational Linguistics (Volume 2: Short Papers), volume 2, pages 75-80.

Roi Blanco, Giuseppe Ottaviano, and Edgar Meij. 2015. Fast and space-efficient entity linking for queries. In Proceedings of the Eighth ACM International Conference on Web Search and Data Mining, pages 179-188. ACM.

Hui Chen, Baogang Wei, Yonghuai Liu, Yiming Li, Jifang Yu, and Wenhao Zhu. 2017. Bilinear joint learning of word and entity embeddings for entity linking. Neurocomputing.

Xiao Cheng and Dan Roth. 2013. Relational inference for wikification. In Proceedings of the 2013 Conference on Empirical Methods in Natural Language Processing, pages 1787-1796.

Kevin Clark and Christopher D Manning. 2016. Deep reinforcement learning for mention-ranking coreference models. In Proceedings of the 2016 Conference on Empirical Methods in Natural Language Processing, pages 2256-2262.

Silviu Cucerzan. 2007. Large-scale named entity disambiguation based on wikipedia data. In Proceedings of the 2007 Joint Conference on Empirical 
Methods in Natural Language Processing and Computational Natural Language Learning (EMNLPCoNLL).

Zheng Fang, Yanan Cao, Qian Li, Dongjie Zhang, Zhenyu Zhang, and Yanbing Liu. 2019. Joint entity linking with deep reinforcement learning. In The World Wide Web Conference, pages 438-447. ACM.

Jun Feng, Minlie Huang, Yijie Zhang, Yang Yang, and Xiaoyan Zhu. 2018. Relation mention extraction from noisy data with hierarchical reinforcement learning. arXiv preprint arXiv:1811.01237.

Matthew Francis-Landau, Greg Durrett, and Dan Klein. 2016. Capturing semantic similarity for entity linking with convolutional neural networks. arXiv preprint arXiv:1604.00734.

Octavian-Eugen Ganea and Thomas Hofmann. 2017. Deep joint entity disambiguation with local neural attention. arXiv preprint arXiv:1704.04920.

Amir Globerson, Nevena Lazic, Soumen Chakrabarti, Amarnag Subramanya, Michael Ringaard, and Fernando Pereira. 2016. Collective entity resolution with multi-focal attention. In Proceedings of the 54th Annual Meeting of the Association for Computational Linguistics (Volume 1: Long Papers), volume 1, pages 621-631.

Zhaochen Guo and Denilson Barbosa. 2016. Robust named entity disambiguation with random walks. Semantic Web, (Preprint):1-21.

Zhengyan He, Shujie Liu, Mu Li, Ming Zhou, Longkai Zhang, and Houfeng Wang. 2013. Learning entity representation for entity disambiguation. In Proceedings of the 51st Annual Meeting of the Association for Computational Linguistics (Volume 2: Short Papers), volume 2, pages 30-34.

Johannes Hoffart, Mohamed Amir Yosef, Ilaria Bordino, Hagen Fürstenau, Manfred Pinkal, Marc Spaniol, Bilyana Taneva, Stefan Thater, and Gerhard Weikum. 2011. Robust disambiguation of named entities in text. In Proceedings of the Conference on Empirical Methods in Natural Language Processing, pages 782-792. Association for Computational Linguistics.

Kristy Hughes, Joel Nothman, and James R Curran. 2014. Trading accuracy for faster named entity linking. In Proceedings of the Australasian Language Technology Association Workshop 2014, pages 3240.

Diederik P Kingma and Jimmy Ba. 2014. Adam: A method for stochastic optimization. arXiv preprint arXiv:1412.6980.

Phong Le and Ivan Titov. 2018. Improving entity linking by modeling latent relations between mentions. arXiv preprint arXiv:1804.10637.
David Milne and Ian H Witten. 2008. Learning to link with wikipedia. In Proceedings of the 17th ACM conference on Information and knowledge management, pages 509-518. ACM.

Vinod Nair and Geoffrey E Hinton. 2010. Rectified linear units improve restricted boltzmann machines. In Proceedings of the 27th international conference on machine learning (ICML-10), pages 807-814.

Karthik Narasimhan, Adam Yala, and Regina Barzilay. 2016. Improving information extraction by acquiring external evidence with reinforcement learning. arXiv preprint arXiv:1603.07954.

Dat Ba Nguyen, Johannes Hoffart, Martin Theobald, and Gerhard Weikum. 2014. Aida-light: Highthroughput named-entity disambiguation. LDOW, 1184.

Thien Huu Nguyen, Nicolas Fauceglia, Mariano Rodriguez Muro, Oktie Hassanzadeh, Alfio Massimiliano Gliozzo, and Mohammad Sadoghi. 2016. Joint learning of local and global features for entity linking via neural networks. In Proceedings of COLING 2016, the 26th International Conference on Computational Linguistics: Technical Papers, pages 23102320.

Jeffrey Pennington, Richard Socher, and Christopher Manning. 2014. Glove: Global vectors for word representation. In Proceedings of the 2014 conference on empirical methods in natural language processing (EMNLP), pages 1532-1543.

Maria Pershina, Yifan He, and Ralph Grishman. 2015. Personalized page rank for named entity disambiguation. In Proceedings of the 2015 Conference of the North American Chapter of the Association for Computational Linguistics: Human Language Technologies, pages 238-243.

Minh C Phan, Aixin Sun, Yi Tay, Jialong Han, and Chenliang Li. 2018. Pair-linking for collective entity disambiguation: Two could be better than all. IEEE Transactions on Knowledge and Data Engineering.

Jonathan Raiman and Olivier Raiman. 2018. Deeptype: Multilingual entity linking by neural type system evolution. arXiv preprint arXiv:1802.01021.

Lev Ratinov, Dan Roth, Doug Downey, and Mike Anderson. 2011. Local and global algorithms for disambiguation to wikipedia. In Proceedings of the 49th Annual Meeting of the Association for Computational Linguistics: Human Language Technologies-Volume 1, pages 1375-1384. Association for Computational Linguistics.

Wei Shen, Jianyong Wang, and Jiawei Han. 2015. Entity linking with a knowledge base: Issues, techniques, and solutions. IEEE Transactions on Knowledge and Data Engineering, 27(2):443-460. 
Wei Shen, Jianyong Wang, Ping Luo, and Min Wang. 2012. Linden: linking named entities with knowledge base via semantic knowledge. In Proceedings of the 21st international conference on World Wide $W e b$, pages 449-458. ACM.

Nitish Srivastava, Geoffrey Hinton, Alex Krizhevsky, Ilya Sutskever, and Ruslan Salakhutdinov. 2014. Dropout: a simple way to prevent neural networks from overfitting. The Journal of Machine Learning Research, 15(1):1929-1958.

Richard S Sutton and Andrew G Barto. 1998. Reinforcement learning: An introduction, volume 1. MIT press Cambridge.

Peng Xu and Denilson Barbosa. 2018. Neural finegrained entity type classification with hierarchyaware loss. north american chapter of the association for computational linguistics, 1:16-25.

Mengge Xue, Weiming Cai, Jinsong Su, Linfeng Song, Yubin Ge, Yubao Liu, and Bin Wang. 2019. Neural collective entity linking based on recurrent random walk network learning. arXiv preprint arXiv:1906.09320.

Ikuya Yamada, Hiroyuki Shindo, Hideaki Takeda, and Yoshiyasu Takefuji. 2016. Joint learning of the embedding of words and entities for named entity disambiguation. arXiv preprint arXiv:1601.01343.

Yi Yang, Ozan Irsoy, and Kazi Shefaet Rahman. 2018. Collective entity disambiguation with structured gradient tree boosting. arXiv preprint arXiv:1802.10229. 